\title{
A computational approach for detecting pigmented skin lesions in macroscopic images
}

\author{
Roberta B. Oliveira $^{a}$, Norian Marranghello ${ }^{b}$, Aledir S. Pereira ${ }^{\mathrm{b}}$, João Manuel R.S. Tavares ${ }^{\mathrm{a}, *}$ \\ a Instituto de Ciência e Inovação em Engenharia Mecânica e Engenharia Industrial, Departamento de Engenharia Mecânica, Faculdade de Engenharia, \\ Universidade do Porto, Rua Dr. Roberto Frias, s/n, 4200-465 - Porto, Portugal \\ ${ }^{\mathrm{b}}$ Departamento de Ciências de Computação e Estatística, Instituto de Biociências, Letras e Ciências Exatas, Universidade Estadual Paulista, Rua Cristóvão \\ Colombo, 2265, CEP: 15054-000, São José do Rio Preto, SP, Brazil
}

\section{A R T I C L E I N F O}

\section{Article history:}

Received 25 January 2016

Revised 10 May 2016

Accepted 11 May 2016

Available online 12 May 2016

\section{Keywords:}

Image pre-processing

Image segmentation

Image classification

Anisotropic diffusion filter

Active contour model without edges

Support vector machine

\begin{abstract}
A B S T R A C T
Skin cancer is considered one of the most common types of cancer in several countries and its incidence rate has increased in recent years. Computational methods have been developed to assist dermatologists in early diagnosis of skin cancer. Computational analysis of skin lesion images has become a challenging research area due to the difficulty in discerning some types of skin lesions. A novel computational approach is presented for extracting skin lesion features from images based on asymmetry, border, colour and texture analysis, in order to diagnose skin lesion types. The approach is based on an anisotropic diffusion filter, an active contour model without edges and a support vector machine. Experiments were performed regarding the segmentation and classification of pigmented skin lesions in macroscopic images, with the results obtained being very promising.
\end{abstract}

(c) 2016 Elsevier Ltd. All rights reserved.

\section{Introduction}

Computational analysis of skin lesion images is an area of great research interest due to its importance in skin cancer prevention, particularly in achieving a successful early diagnosis (Celebi et al., 2007b; Filho, Ma, \& Tavares, 2015; Scharcanski \& Celebi, 2013). Such lesions, which can be classified as benign or malignant, are mainly due to abnormal production of melanocyte cells originating from factors such as excessive sun exposure. Melanocyte cells are responsible for creating the substance melanin, whose main function is to provide skin pigmentation. In the case of malignant cells, i.e. melanoma (Fig. 1a), such cells divide quickly and may invade other parts of the body. An increasing number of deaths due to melanoma have been observed worldwide, since this type of malignant lesion is the most aggressive compared to other lesion types due to its high level of metastasis (INCA, 2014). Benign lesions display a more organized structure than malignant lesions, since the former are unable to proliferate into other tissues. Seborrheic keratosis (Fig. 1b) and melanocytic nevus (Fig. 1c) are examples of benign lesions. However, these skin lesions have

* Corresponding author. Fax: +351 225081445.

E-mail addresses: roberta.oliveira@fe.up.pt (R.B. Oliveira), norian@ibilce.unesp.br (N. Marranghello), aledir@ibilce.unesp.br (A.S. Pereira), tavares@fe.up.pt (J.M.R.S. Tavares).

URL: http://www.fe.up.pt/ tavares (J.M.R.S. Tavares) also been of global concern, since some types of nevi may become melanoma; moreover a melanoma may resemble a seborrheic keratosis or a nevus in its initial state.

Different non-invasive imaging techniques have been employed to assist dermatologists in diagnosing skin lesions (Smith \& MacNeil, 2011). Macroscopic images, commonly known as clinical images, are normally used in computational analysis of skin lesions (Cavalcanti \& Scharcanski, 2013; Wong, Scharcanski, \& Fieguth, 2011), since such images may be obtained using common digital video or image cameras. Fig. 1 presents examples of macroscopic images. However, their imaging conditions are frequently inconsistent; for example, images are acquired from variable distances and/or under different illumination conditions. Furthermore, the images may have poor resolution, which may be challenging when the lesion under study is small. An additional problem with clinical images is related to the presence of artefacts, such as hair, reflections, shadows and skin lines, which may hinder adequate analysis of the imaged skin lesions.

Pre-processing, segmentation, feature extraction, and classification are fundamental steps commonly found in computational systems of image analysis. In terms of skin lesions, the image pre-processing step is an important aspect for good segmentation, i.e. identification, of the image's pigmented skin lesions. Effective approaches based on colour space transformation (Abbas, Celebi, \& Garcia, 2012a), contrast enhancement 


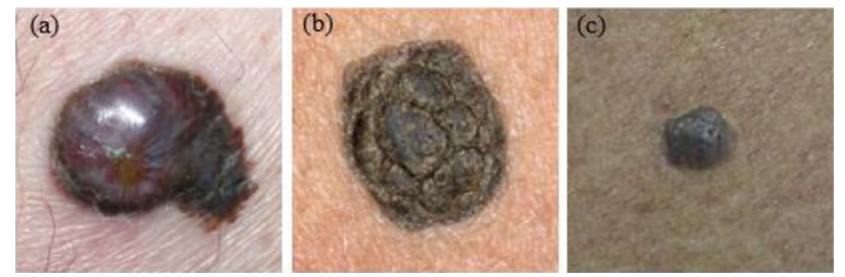

Fig. 1. Three examples of pigmented skin lesions: (a) melanoma, (b) seborrheic keratosis and (c) melanocytic nevus.

(Schaefer, Rajab, Celebi, \& Iyatomi, 2011) and artefact removal (Abbas, Garcia, Celebi, Ahmad, \& Mushtaq, 2013b) have been proposed for this step in order to improve the accuracy of the following segmentation step. Segmentation allows for extracting the region of interest (ROI) from the macroscopic image under analysis. Previous studies (Silveira et al., 2009; Wong et al., 2011; Zhou et al., 2010) have shown that computational methods for image segmentation may provide suitable results for the identification of skin lesions in images.

The feature extraction of skin lesion images is usually based on methods used by dermatologists in their clinical routine diagnosis. Of these methods, the $A B C D$ rule is mostly used, being a criteria based on the Asymmetry, Border, Colour and Diameter characteristics of the lesion under study (Abbasi et al., 2004). The asymmetry criterion may be examined by dividing the region of the lesion into two sub-regions by an axis of symmetry, in order to analyse the similarity of the area by overlapping the two sub-regions along the axis. The lesion is considered symmetric when the two subregions are highly similar, which is prevalent in benign lesions. Otherwise, the lesion is considered asymmetric which is associated with malignant lesions. The border criterion corresponds to the measure of the regularity of the lesion's shape. According to this criterion, a border of regular shape is associated with benign lesions while a border of irregular shape is associated with malignant lesions instead. The colour criterion consists of analysing the tonality variation of the pigmented skin lesions in order to identify the malignant lesions, which usually present non-uniform colours. The diameter criterion is associated with the size of the lesion and is defined by the greatest distance between any two points of the lesion's border. As such, a diameter equal to or greater than 6 (six) millimetres may indicate malignancy. Texture analysis may also be performed for image-based examination of skin lesions, since it assists in discriminating benign from malignant lesions by assessing the roughness of their structure (Cavalcanti \& Scharcanski, 2013).

Several computational solutions (Celebi et al., 2007b; Iyatomi, et al., 2010) have been proposed for extracting features from pigmented skin lesions in images in order to represent them according to certain criteria. Then, the classification step consists of recognizing and interpreting the information about the pigmented skin lesions based on these features. Hence, computational classifiers are important tools to assist the computational diagnosis of skin lesions in macroscopic images (Celebi et al., 2008a; Iyatomi, et al., 2008; Maglogiannis \& Doukas, 2009).

The objective of this work was to develop a novel computational approach based on the $\mathrm{ABCD}$ rule and texture analysis for the identification and classification of pigmented skin lesions in macroscopic images, in order to provide information that may assist dermatologists in their diagnosis. In this approach, an anisotropic diffusion filter (Barcelos, Boaventura, \& Silva, 2003) is applied to reduce the noise present in the image under study. Then, the active contour model without edges (Chan \& Vese, 2001) is employed in the segmentation of the lesion in the pre-processed image. Afterwards, features related to the asymmetry, border, colour and texture of the lesion are extracted from the segmented image. Finally, the features are used as input to a support vector machine (SVM) classifier (Burges, 1998) to classify the skin lesion.

This paper is organized as follows: a review of the computational methods that have been applied to classify pigmented skin cancers and other skin lesions is provided in Section 2. A novel approach for detecting and classifying skin lesions in dermoscopy images is proposed in Section 3. The results and their discussion are provided in Section 4. Finally, conclusions drawn and proposal for future studies are in the last section.

\section{Related studies}

Computer-aided diagnosis (CAD) systems for skin lesions in images have been proposed in order to assist dermatologists, predominantly in the early assessment of skin cancer. In these systems, image filters are commonly applied to pre-process the input images in order to increase the accuracy of the segmentation step. A median filter, which is a non-linear image filtering algorithm, has been applied often to smooth images of skin lesions as well as to remove artefacts, preserving the border of the lesion, which is imperative to assure adequate segmentation ( Celebi et al., 2007b, 2008b; Silveira et al., 2009). An anisotropic diffusion filter has also been regularly used for smoothing skin lesion images, particularly to remove artefacts with good results and without losing relevant information about lesions (Barcelos \& Pires, 2009). Based on set theory, morphological filtering (Gonzalez \& Woods, 2002) enables removing image noise (Norton et al., 2010; Silveira et al., 2009), and may also be used to enhance skin lesions in images (Beuren, Janasieivicz, Pinheiro, Grando, \& Facon, 2012), as well as to include areas with borders of low contrast in previously detected lesion regions ( Norton et al., 2010, 2012).

Algorithms of image segmentation have been developed based on several techniques to assist the diagnosis of skin lesions from images (Oliveira et al., 2016). From these, threshold-based algorithms have been widely used, mainly because of their simplicity. Thus, thresholding algorithms, such as the Otsu (Celebi et al., 2007b; Celebi, Wen, Hwang, Iyatomi, \& Schaefer, 2013; Norton et al., 2010, 2012), type-2 fuzzy logic (Yuksel \& Borlu, 2009) and the Renyi entropy method (Beuren et al., 2012), aim to establish the threshold values in order to separate the regions of interest (ROIs) in the input images. However, these techniques may reveal some problems; for example the segmented lesions tend to be smaller than their real size, and the segmentation process may lead to highly irregular lesion borders (Yuksel \& Borlu, 2009).

Algorithms based on active contour models (ACM) have been frequently proposed for the segmentation of skin lesions in images (Abbas et al., 2012a; Silveira et al., 2009; Zhou et al., 2010). In these algorithms, initial curves move toward the boundaries of the objects of interest through appropriate deformation. The algorithms of active contour may be classified as edge- or regionbased models (Zhang, Song, \& Zhang, 2010) according to the technique used to track the curves movement. Additionally, mixed models have been also adopted, see, for example, Li, Xu, Gui, and Fox (2010). The edge-based models include classic parametric models (Kass, Witkin, \& Terzopoulos, 1988), gradient vector flow (GVF) (Xu \& Prince, 1998) and geometric (or geodesic) active contours (GAC) (Paragios \& Deriche, 2002). However, classic parametric models and GVF have difficulty in dealing with topological changes and large curvatures. On the other hand, GAC models, such as level-set-based algorithms, do not present such problems. The region-based active contour model proposed by Chan and Vese (2001) has been used in the segmentation of skin lesions (Silveira et al., 2009) due to its advantages relatively to other segmentation algorithms based on ACM (Chan \& Vese, 2001), such as: (1) the initial curve may be defined more freely in the input image, (2) the inner contours are automatically detected without the 
need to define additional curves in the image, and (3) the segmentation is successfully carried out even in the presence of intensity variations, very smooth boundaries and boundaries not successfully detected by gradient operators. Region-based algorithms, like the region growing, splitting and merging methods have also been used to segment skin lesions in images (Celebi et al., 2007a, 2007b; Iyatomi et al., 2008; Silveira et al., 2009). These methods consist of grouping similar neighbouring pixels, or sub-regions, into larger homogeneous regions according to a growing criterion. Such methods have shown successful performance even under complex conditions such as great variations of illumination and colour. However, some of these methods may not adequately identify lesion regions that present low contrast relatively to the skin background.

The wide use of algorithms based on artificial intelligence (AI) is justified by the advantages they offer (Silveira et al., 2009), such as the possibility of learning from sample cases provided by artificial neural networks (ANNs) (Schaefer et al., 2011), the search and optimization for the best segmentation results provided by techniques based on genetic algorithms (GAs) (Roberts \& Claridge, 2003), and the capability of dealing with imprecise values provided by fuzzy logic, e.g., by applying the type-2 fuzzy logic technique (Yuksel \& Borlu, 2009). In addition, fuzzy logic combined with clustering techniques have been employed in the image segmentation of skin lesions, such as the fuzzy c-means (FCM) algorithm (Rahman, Bhattacharya, \& Desai, 2008) and the anisotropic mean shift approach based on the FCM algorithm (AMSFCM) (Zhou, Schaefer, Sadka, \& Celebi, 2009). The hill-climbing algorithm (HCA) is a technique based on the clustering of points on an image, which is also applied to detect ROIs in skin lesion images (Abbas, Garcia, Celebi, Ahmad, \& Mushtaq, 2013a). In Abbas, Celebi, and García (2012b), a new segmentation method based on dynamic programming was proposed to overcome the limitation of thresholding, region-growing and clustering, as well as level-set-based segmentation methods. However, some algorithms based on AI may also present disadvantages regarding the complexity of their implementation and the presence of unnecessary steps, which requires high computational efforts (Roberts \& Claridge, 2003).

The ABCD rule and texture analysis are examples of approaches employed in the literature for the computational analysis of skin lesions in macroscopic images. However, other descriptors have also been extracted for the characterization of skin lesions in images:

- Asymmetry (A): asymmetry index descriptors based on axis of symmetry (Chang, Stanley, Moss, \& Van Stoecker, 2005; She, Liu, \& Damatoa, 2007), and geometrical descriptors (Cavalcanti \& Scharcanski, 2013);

- Border (B): geometrical descriptors based on the best-fit of ellipse axes (Chang et al., 2005; She et al., 2007), and statistical descriptors based on border gradient and edge regions (Cavalcanti \& Scharcanski, 2013);

- Colour (C): statistical descriptors based on colour models (Cavalcanti \& Scharcanski, 2013; Chang et al., 2005; She et al., 2007), amount of colour pixels (Cavalcanti \& Scharcanski, 2013), and relative colour descriptors (Chang et al., 2005);

- Diameter (D): semi-major axis of the best-fit ellipse (She et al., 2007); and

- Texture $(\mathrm{T})$ : statistical descriptor based on the intensity of the pixels inside the lesion regions (Cavalcanti \& Scharcanski, 2013).

For the classification process, one or several methods have been evaluated to achieve the best results. The performance of this process depends on several issues, such as the quality of the segmented image and extracted features, as well as on the classification method used. The output of the skin lesion classification process may be binary or multi-class, and concern different classes according to the classification goal, e.g., malignancy of the lesions
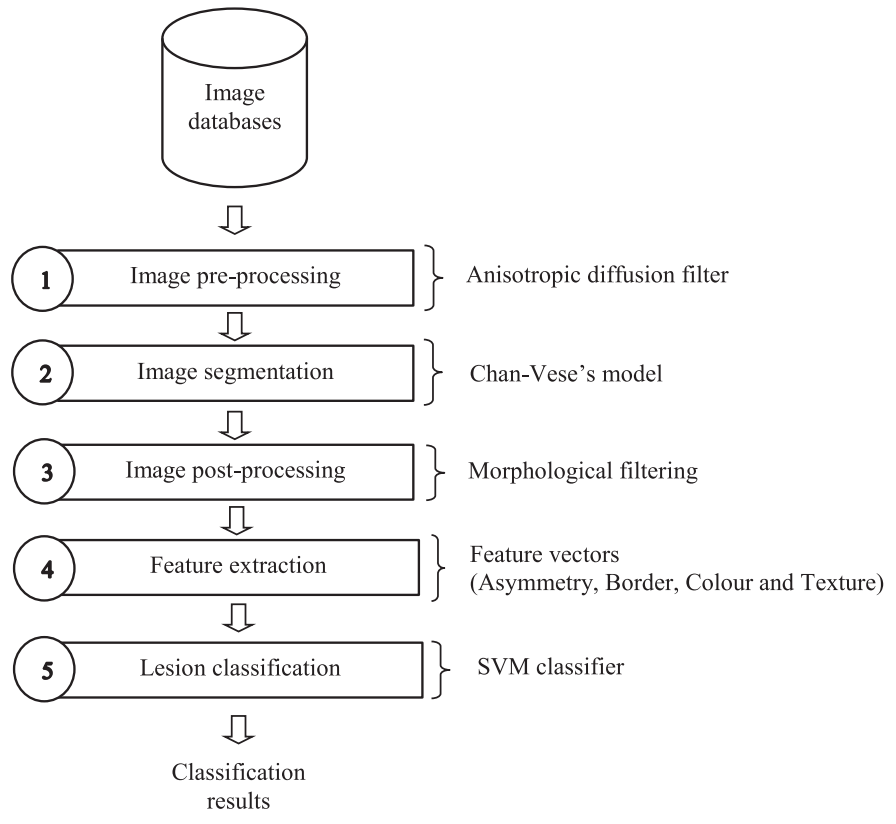

Fig. 2. Pipeline of the proposed approach for detecting and classifying pigmented skin lesions in images.

(benign versus malignant) (Garnavi, Aldeen, \& Bailey, 2012), and distinct types of skin lesions (melanoma versus nevus (Iyatomi et al., 2008; Maglogiannis \& Doukas, 2009), melanocytic versus non-melanocytic (Iyatomi, et al., 2010), and dysplastic versus nondysplastic versus melanotic (Maglogiannis \& Doukas, 2009). Furthermore, skin lesion features, such as border features regular versus irregular (Clawson, Morrow, Scotney, McKenna, \& Dolan, 2009) can also be classified.

Classification methods based on a decision tree have been used in the classification of skin lesions by many authors (Celebi et al., 2008a; Chang et al., 2005; Maglogiannis \& Doukas, 2009). The simplicity of the classification structure in terms of ease of understanding and visualization, as well as the easy rule generation, is one of the important advantages of this approach. However, the difficulties in dealing with correlated features and the possibility of excessive adjustments (over-fitting) are its major drawbacks. Bayesian learning-based methods have also been applied to classify skin lesions (Garnavi et al., 2012; Maglogiannis \& Doukas, 2009). Although Bayesian methods provide fast training and no sensitivity to irrelevant features, they assume that the features are independent. Despite the long training time, artificial neural networks have been proposed in various studies (Iyatomi et al., 2008; Maglogiannis \& Doukas, 2009) to cope with many complex pattern recognition problems, since such classifiers present good capability and flexibility to solve several non-separable problems. The SVM classifier (Burges, 1998) has also been applied to discriminate skin lesions, due to its good generalization and simplification of the nonlinear data separation by means of kernel functions (Celebi et al., 2007b; Maglogiannis \& Doukas, 2009). The SVM performed better than other computer classifiers in several studies (Maglogiannis \& Doukas, 2009). However, this classifier may be sensitive to noise and the classification process is binary.

\section{Proposed approach}

In this section, a computational approach for identification and classification of pigmented skin lesions in macroscopic images is presented, in order to provide information that may assist dermatologists in their diagnosis. Fig. 2 illustrates the pipeline of the proposed approach, which involves the following steps: (1) image 
pre-processing, (2) image segmentation, (3) image post-processing, (4) feature extraction, and (5) lesion classification. The first step is mainly applied to deal with noisy images based on an anisotropic diffusion filter (Barcelos et al., 2003). The second step is responsible for identifying the lesion presented in the image being studied by using an active contour model without edges, known as Chan-Vese's model (Chan \& Vese, 2001). The third step consists of the post-processing of the segmented image based on morphological filtering (Gonzalez \& Woods, 2002) in order to improve the quality of the segmentation result. In the fourth step, features are extracted from the identified lesion, including the lesion's asymmetry, border, colour and texture properties. Finally, the last step concerns the lesion classification based on the extracted features that are inputted into an SVM classifier (Burges, 1998). In the next sections, each step of the proposed approach is detailed.

\subsection{Image pre-processing}

As mentioned previously, the image under analysis may contain several artefacts that can affect the accuracy of the image segmentation step. Hence, an anisotropic diffusion filter (Barcelos et al., 2003 ) is applied to smooth the input image, mainly in order to reduce the presence of hairs. Hence, initially, the original RGB (red, green, blue) image is converted into a grey-level image, since the segmentation method used is applied to grey-level images. Afterwards, the anisotropic diffusion filter is applied to the converted image according to the solution proposed by Barcelos et al. (2003), which aims at smoothing very noisy images without removing relevant borders.

The implementation of this filter is based on the following equations:

$u_{t}=g\left(\left|\left(G_{\sigma} * \nabla u \mid\right)\right| \nabla u \mid \operatorname{div}\left(\frac{\nabla u}{|\nabla u|}\right)-\lambda(1-g)(u-I)\right.$,

$g\left(\mid\left(G_{\sigma} * \nabla u \mid\right)=\frac{1}{1+k\left|\left(G_{\sigma} * \nabla u\right)\right|^{2}}\right.$, and

$G_{t}(x, y)=\frac{1}{2 \pi \sigma t^{2}} e^{\frac{-\left|x^{2}+y^{2}\right|}{2 \sigma t^{2}}}$,

where $u(x, y, 0)=I(x, y), x \in \Omega, t>0, I(x, y)$ is the original image to be processed, $u$ the smoothed image at scale $t$, div the divergence operator, $\nabla u$ the gradient of $u$, and $\lambda$ a parameter related to the diffusion speed. The term $g\left(\mid\left(G_{\sigma} * \nabla u \mid\right)\right.$ is used for border detection, where $k$ is a parameter, $G_{\sigma}$ the Gaussian function, and $\sigma$ the standard deviation of $G_{\sigma}$. The convolution $G_{\sigma} * \nabla u$ is a Gaussian scale space of $g$ given by: $T_{g}(x, y, t)=g_{*} G_{t}(x, y)$ where $G_{t}$ is given by Eq. (3) and $t$ is the scale. Considering a neighbourhood of a pixel $x$, when the gradient $\nabla$ has a low average value; i.e., there are few noisy pixels in the input image, $x$ is considered an inner pixel (homogeneous region), resulting in $g \cong 1$. Otherwise, $x$ will be a pixel of a contour, $g \cong 0$. The moderation selector $(1-g)$ (Barcelos et al., 2003) allows a balanced diffusion of the input image, i.e., the homogeneous regions are smoothed even more with respect to the borders of the regions. This filter is iteratively applied to the image, such that the number of iterations $(N I)$ is determined according to the amount of noise presented in the input image. However, relevant borders may be removed when the number of iterations is too large.

\subsection{Image segmentation}

The segmentation process should be effective, so information of the lesion may be extracted with high confidence. In addition, the accuracy of this process directly influences the feature extraction step, which is required to suitably represent the lesion for its classification process. Therefore, an appropriate segmentation technique is crucial to obtain good classification results for the problem in question. The Chan-Vese model (Chan \& Vese, 2001) is based on the average of the intensities of the image's pixels, and not on the image's gradient. This model uses the concepts of the MumfordShah and level-set segmentation models. Essentially, Chan-Vese's model considers a "fitting" term $F$ for the energy minimization, which allows the deformation of the curve toward the boundary of the object to be segmented, in which the inside and outside intensities are constant and similar. In order to identify whether the object of interest is inside or outside the curve, the energy minimization $F\left(c_{1}, c_{2}, \phi\right)$ is calculated as:

$$
\begin{aligned}
F\left(c_{1}, c_{2}, \phi\right)= & \mu \int_{\Omega} \delta(\phi(x, y))|\nabla \phi(x, y)| d x d y+v \int_{\Omega} H(\phi(x, y)) d x d y \\
& +\lambda_{1} \int_{\Omega}\left|u_{0}(x, y)-c_{1}\right|^{2} H(\phi(x, y)) d x d y \\
& +\lambda_{2} \int_{\Omega}\left|u_{0}(x, y)-c_{2}\right|^{2}(1-H(\phi(x, y))) d x d y, \quad(4)
\end{aligned}
$$

where $u_{0}$ is a pre-processed image, as a bounded function on $\bar{\Omega}$ and with real values. The fixed parameters $\mu, v \geq 0$, and $\lambda_{1}$ and $\lambda_{2}>0$ are weights for the fitting term. The terms $H$ and $\delta$ are the Heaviside and Dirac delta functions, respectively, used in order to obtain the level-set energy function $F\left(c_{1}, c_{2}, \phi\right)$. The constants $c_{1}$ and $c_{2}$, which are based on the Mumford-Shah segmentation model, are the average image $u_{0}$ inside and outside curve $C$, respectively. Such constants are given by:

$$
\begin{aligned}
& c_{1}(\phi)=\frac{\int_{\Omega} u_{0}(x, y) H(\phi(x, y)) d x d y}{\int_{\Omega} H(\phi(x, y)) d x d y}, \\
& c_{2}(\phi)=\frac{\int_{\Omega} u_{0}(x, y)(1-H(\phi(x, y))) d x d y}{\int_{\Omega}(1-H(\phi(x, y))) d x d y}
\end{aligned}
$$

\subsection{Image post-processing}

Frequently, the segmentation results are post-processed to improve the accuracy of the obtained lesion region. In many cases, morphological operations (Gonzalez \& Woods, 2002) are employed for this purpose (Celebi et al., 2008b; Norton et al., 2012; Zhou et al., 2009). Here, a morphological filtering, presented in Eq. (7), is applied to the segmented image $I$ by using a structuring element $E$. This process allows the smoothing of borders, the removing of isolated regions, and/or even filling the segmented lesion region. This filter consists of the opening operation $I \circ E$, defined by Eq. (8), followed by the closing operation of the result by $E$, defined by Eq. (9), respectively:

$(I \circ E) \cdot E$,

$I \circ E=(I \ominus E) \oplus E$,

$I \cdot E=(I \oplus E) \ominus E$,

where $I \oplus E$ is the dilation operation given by Eq. (10) and $I \ominus E$ is the erosion operation given by Eq. (11). Therefore, the opening of set $I$ by $E$ is the erosion of $I$ by $E$, followed by the dilation of the result by $E$. The closing of the set $I$ by $E$ is the dilation of $I$ by $E$, followed by the erosion of the result by $E$ :

$I \oplus E=\left\{x \mid\left[(\hat{E})_{x} \cap I\right] \subseteq I\right\}$,

$I \ominus E=\left\{x \mid(E)_{x} \subseteq I\right\}$,

where $\hat{E}$ is the reflection of set $E$ (structuring element), $(\hat{E})_{X}$ is the translation of set $\hat{E}$ by pixel $x$, and $(E)_{x}$ is the translation of set $E$ by pixel $x$. 


\subsection{Feature extraction}

After the ROI identification, the next step is to extract a lesion's features based on the $A B C D$ rule in order to numerically describe its properties. The clinical assessment is usually based on all of the rule's criteria to diagnose the malignancy of lesions in images. However, the diameter criterion was not applied here due to its great dependence on the image resolution (Celebi et al., 2007b), since the size of the image highly affects the number of pixels of each segmented lesion regions. Instead, a texture analysis is performed to assess the surface roughness of the lesion. Therefore, asymmetry, border, colour and texture properties are extracted from the original RGB image using the segmented image after post-processing as a feature extraction mask.

\subsubsection{Asymmetry}

In order to extract features based on the asymmetry criterion, the region of the lesion under analysis is dividing into two subregions $\left(R_{1}, R_{2}\right)$ by an axis according to the longest diagonal $d$ defined by Euclidian distance (Gonzalez \& Woods, 2002):

$D_{(p, q)}=\sqrt{\left(x_{1}-x_{2}\right)^{2}+\left(y_{1}-y_{2}\right)^{2}}$,

where $\left(x_{1}, y_{1}\right)$ and $\left(x_{2}, y_{2}\right)$ are the coordinates of the border's pixels $p$ and $q$. All the border's pixels are analysed in order to find which pair has the greatest distance $D_{(p, q)}$.

Perpendicular lines from the pixels of the longest diagonal $d$ are computed to analyse the similarity between two sub-regions of the lesion. The number of perpendicular lines may be different for each image, since it depends on the size of the diagonal $d$ of the lesion. Therefore, $N=T / P$ is computed to determine the number of perpendicular lines for all images to be classified; i.e. it determines a set of perpendicular lines $S$, where $T$ is the total number of perpendicular lines along the diagonal $d$, and $P$ is a pre-defined fixed number of expected perpendicular lines. In order to determine an adequate set $S$, the following values for $P$ have been experimentally established, $P=\{10,20,30,40,50\}$. Ten perpendicular lines $P=10$ obtained the best results in experimental tests to represent the size of the set of perpendicular lines for each image. Afterwards, two semi-lines were determined from each perpendicular line of the set $S$, one semi-line represents the sub-region $R_{1}$, and the other represents the sub-region $R_{2}$. For each perpendicular, the distance $D_{(p, q)}$ of the semi-line for both sub-regions $\left(R_{1}, R_{2}\right)$ is computed, where $p$ is a pixel of the diagonal $d$ and $q$ is a pixel of the border. rion:

Eleven features are extracted to represent the asymmetry crite-

- The ratio between the shortest and longest distances based on the semi-lines $\left(R_{1}, R_{2}\right)$ from each perpendicular line of set $S$ (10 features);

- The standard deviation from ratios based on all perpendicular lines of set $S$ (1 feature).

The ratio between the two semi-lines allows for determining whether the lesion area may be more symmetric or more asymmetric to a particular pixel of the longest diagonal, i.e., the area is either more asymmetric when its coefficient is closer to zero, or more symmetrical when its coefficient is closer to one.

\subsubsection{Border}

A border is represented by pixels comprising the lesion's boundary, obtained as a result of the lesion segmentation process. A one-dimensional border (Gonzalez \& Woods, 2002) of the lesion under analysis is defined to extract features based on this criterion. The number of peaks, valleys and straight lines of the border is extracted by vector product and inflexion point descriptors by means of the one-dimensional border. An inflexion point descriptor is applied to measure small irregularities in the border, whereas a vector product descriptor is applied to measure substantial irregularities in the border (Araujo, 2010).

The inflexion point descriptor aims to analyse border's pixels $P_{i}$ to define which pixels show a change of direction. Therefore, a four-point neighbourhood $N_{j}$ for both left and right directions is considered for each border's pixel $P_{i}$. In order to detect if a given pixel $P_{i}$ is an inflexion, weights $w_{j}$ are assigned to its neighbour pixels. From the analysis of the $y$ axis of a system of coordinates, each neighbour pixel $N_{j}$ that is below the pixel under analysis $P_{i}$ receives $w_{j}=1$. Otherwise, each neighbour pixel receives $w_{j}=-1$. Afterwards, the weights $w_{j}$ corresponding to each direction (left, $D_{l}$, and right, $D_{r}$ ) are added separately, $D_{l}, D_{r}=\sum_{j} w_{j}$. Pre-defined thresholds $T_{1}=2$ and $T_{2}=-2$ (Araujo, 2010) are considered to analyse small irregularities in the border, based on the sum of the weights $D_{l}, D_{d}$. An inflexion pixel $P_{i}$ is achieved when $D_{l}$ and $D_{r} \geq T_{1}$ or $D_{l}$ and $D_{r} \leq T_{2}$. The sum of the weights for both left and right neighbour pixels $S_{i}=D_{l}+D_{r}$ identifies the inflexion pixel $P_{i}$ as a peak when $S_{i}>0$, as a valley when $S_{i}<0$, or as a straight line when $S_{i}=0$.

On the other hand, the vector product descriptor aims to analyse a border's pixels to identify peaks and valleys with substantial irregularities. The vector product $V_{i}$ is based on three border pixels $p_{1}, p_{2}$, and $p_{3}$ established according to a difference of fifteen pixels between them, totalling a difference of thirty pixels between $p_{1}$ and $p_{3}$ (Araujo, 2010). The vector product $V_{i}$ is computed for each border's pixels as:

$V_{i}=\left(x_{2}-x_{1}\right)\left(y_{3}-y_{1}\right)-\left(y_{2}-y_{1}\right)\left(x_{3}-x_{1}\right)$,

where $\left(x_{1}, y_{1}\right),\left(x_{2}, y_{2}\right)$ and $\left(x_{3}, y_{3}\right)$ are the three aforementioned pixels $p_{1}, p_{2}$, and $p_{3}$. Such points determine whether a segment belongs to a peak, valley or straight line. Therefore, a border's pixel $P_{i}$ is identified as a peak when $V_{i}>0$, as a valley when $V_{i}<0$, or as a straight line when $V_{i}=0$.

Six features are extracted to represent the border criterion:

- The number of peaks, valleys and straight lines based on small irregularities of the border by using the inflexion point descriptor (3 features);

- The number of peaks, valleys and straight lines based on large irregularities of the border by using the vector product descriptor (3 features).

The peak, valley and straight-line values may be relatively different for each image, since they depend on the size of the lesion's border. In order to solve the problem of different ranges that may influence the classification results, such values are adjusted into an interval between 0 (zero) and 1 (one). Therefore, the values obtained by the inflexion point descriptor are divided by the total number of pixels obtained, and the values obtained by the vector product descriptor are divided by the total number of border's pixels. These features allow the assessment of the regularity or irregularity of the lesion's border.

\subsubsection{Colour}

The $R G B$ colour model is commonly employed to represent the colours of skin lesions in images (Celebi et al., 2007b; Chang et al., 2005; Iyatomi et al., 2008, 2010; She et al., 2007). Therefore, statistical measures based on this model are applied to represent the colour criterion. The mean, variance and standard deviation values for each RGB channel were extracted (nine features). These features allow for analysing tonality changes of pigmented skin lesions in order to identify malignant lesions. 


\subsubsection{Texture}

In order to extract texture properties of the skin lesions, fractal dimensions are computed from the image under study by using a box-counting method (BCM), since it is simple and effective (Dobrescu, Dobrescu, Mocanu, \& Popescu, 2010; Garnavi et al., 2012). A fractal dimension (Al-Akaidi, 2004) is a procedure for splitting the input image into several quadrants to quantify the irregularity level or self-similarity of the image's fractals according to:

$D=\frac{\log (\mathrm{N})}{\log (1 / \mathrm{T})}$

where $\mathrm{N}$ represent the number of elements of the self-similar parts that reconstruct the original image, and $\mathrm{T}$ is the amount of quadrants corresponding to a fraction of its previous size.

The BCM method demarcates a grid over the image; i.e., it divides the image into several squares. The process is iterative, in which the size of each square decreases and the amount of squares that covered the fractal is counted at each iteration. The boxcounting algorithm uses a least squares error to compute the fractal dimension:

$e=\sum_{i}\left(f_{i}-\widehat{f}_{i}\right)^{2}$, with $i=1,2, \ldots, N$,

where $N$ is the number of elements and the term $\widehat{f}_{i}$, which is an approximation to function $f_{i}$, is defined as:

$\widehat{f}_{i}=\beta x_{i}+c$,

where the slope $\beta$ and intercept $c$ of the line $\widehat{f}_{i}$ are computed as:

$\beta=\frac{N \sum_{i} f_{i} x_{i}-\left(\sum_{i} f_{i}\right)\left(\sum_{i} x_{i}\right)}{N \sum x_{i}^{2}-\left(\sum_{i} x_{i}\right)^{2}}$,

$c=\frac{\sum_{i} f_{i}-\beta \sum_{i} x_{i}}{N}$

The image-based fractal dimension $D_{2}$ is computed individually for each row and column of the image. Afterwards, the final fractal dimension is defined as:

$D_{2}=\left(\frac{\sum_{i} D_{i}}{t}\right)+1$

where $D_{i}$ is the fractal dimension obtained at each iteration and $t$ is the total amount of fractal dimensions.

Eighteen features are extracted to represent the texture properties of the lesion under analysis:

- The fractal dimension of the lesion's area (1 feature);

- The fractal dimension of the original image (1 feature);

- The fractal dimension of sixteen parts of the image, with the original image divided into parts of the same size to measure their fractal dimension (16 features).

The fractal dimension is a value between two and three, which allows for measuring the irregularity level or self-similarity of the image surface.

Overall, the number of features $m$ extracted from each image under study is 44 (11 asymmetry, 6 border, 9 colour and 18 texture features). From this set of features, datasets are constructed with a set of samples $\left(x_{\mathrm{i}}\right)$, according to the number of images $n$ for a given classification problem, $i=1, \ldots, n$. Each sample $\left(x_{\mathrm{i}}\right)$ is composed of $m$ features $\left(x_{i m}\right)$ and the class to which it belongs $\left(y_{i}\right)$. Such datasets are used for the classification process.

\subsection{Lesion classification}

After building the set of features, the next step is the lesion classification based on the extracted features. The classification process occurs by randomly dividing the available image samples into training and test sets. The training step consists of developing a classification model based on the training samples, which are applied as input data to the classifier for the learning process. The testing step consists of measuring the accuracy of the model learned in the training step over the set of tests. The classification process should have high performance and robustness, since its results are often used to assist dermatologists in their diagnosis. Therefore, the SVM classifier (Burges, 1998) was used mainly due to its good generalization properties.

The SVM classifier involves an algorithm based on statistical learning applied to build a hyperplane that separates the data according to the defined classes. Such data may be linearly separable or linearly non-separable. Let us consider the training data $\left\{x_{i}\right.$, $y_{i}$, with $x_{i} \in \mathrm{X}$ and $y_{i} \in \mathrm{Y}$, where $\mathrm{X}$ is the set of samples and $\mathrm{Y}$ is the class to which they belong $\{-1,+1\}$. A separating hyperplane may be defined as $f(x)=w \cdot x+b$. Then, the points $x$ that lie on the hyperplane satisfy $f(x)=0$, where $w$ is the normal distance to the hyperplane, and $|b| /\|w\|$ is the perpendicular distance from the hyperplane to the origin, with $b \in \Re$ and $\|w\|$ being the Euclidian norm of $w$. Therefore, the $f(x)$ divides $\mathrm{X}$ into two regions: positive samples if $f(x)>0$, and negative samples if $f(x)<0$. For the linearly separable case, the algorithm is used to search the data with largest distance ("named as largest margin") from the hyperplane based on the following constraints:

$y_{i}\left(w \cdot x_{i}+b\right)-1 \geq 0$, with $\forall_{i}=1, \ldots, n$,

where $w \cdot x_{i}+b \geq+1$ for $y_{i}=+1$, and $w \cdot x_{i}+b \leq-1$ for $y_{i}=-1$.

The largest border is represented by a pair of parallel hyperplanes, $\mathrm{H}_{1}$ and $\mathrm{H}_{2}$. The points defined for these hyperplanes are the training points used for classification, called support vectors. The pair of hyperplanes is obtained by minimization of $\|w\|^{2}$ based on the constraints defined in Eq. (20). Such minimization is given by the Lagrangian function subject to the conditions $w=$ $\sum_{i=1}^{n} \alpha_{i} y_{i} x_{i}$ and $\sum_{i=1}^{n} \alpha_{i} y_{i}=0$, where $\alpha_{i}$ are positive Lagrange multipliers for each of the constraints (Eq. (20)). The Lagrangian function is defined as:

$L_{1}=\sum_{i=1}^{n} \alpha_{i}-\frac{1}{2} \sum_{i, j=1}^{n} \alpha_{i} \alpha_{j} y_{i} y_{j} x_{i} x_{j}$, with $\alpha_{i} \geq 0$.

For the linearly non-separable case, positive slack variables $\xi_{i}$, $i=1, \ldots, n$, are introduced in the constraints:

$y_{i}\left(w \cdot x_{i}+b\right) \geq 1-\xi_{i}$, with $\xi_{i} \geq 0$, and $\forall_{i}=1, \ldots, n$,

where $w \cdot x_{i}+b \geq+1-\xi_{i}$ for $y_{i}=+1$, and $w \cdot x_{i}+b \leq-1+\xi_{i}$ for $y_{i}=-1$. In order to deal with noise and outliers, parameter $C$ is introduced for assigning a penalty to errors, which becomes:

$L_{2}=\sum_{i=1}^{n} \alpha_{i}-\frac{1}{2} \sum_{i, j=1}^{n} \alpha_{i} \alpha_{j} y_{i} y_{j} x_{i} x_{j}$

subject to $0 \leq \alpha_{i} \leq C, \forall_{i}=1, \ldots, n$, and $\sum_{i=1}^{n} \alpha_{i} y_{i}=0$.

In order to simplify the process of separating the non-linear data, a kernel function may be applied to map the set of samples of the original space $\mathrm{X}$ to a new space with infinite dimensional $\Im$, defined as $\Phi: X \rightarrow \Im$. The kernel function receives two points of the original space $\left(\mathrm{x}_{i}, \mathrm{x}_{j}\right)$, and computes the scalar product in the new space, defined as $K\left(\mathrm{x}_{i}, \mathrm{x}_{j}\right)=\Phi\left(\mathrm{x}_{i}\right) \cdot \Phi\left(\mathrm{x}_{j}\right)$. The mapping, by using kernel function based on a dual problem presented in Eq. (23), is defined as:

$L_{3}=\sum_{i=1}^{n} \alpha_{i}-\frac{1}{2} \sum_{i, j=1}^{n} \alpha_{i} \alpha_{j} y_{i} y_{j} K\left(\mathrm{x}_{i}, \mathrm{x}_{j}\right)$, 
subject to $0 \leq \alpha_{i} \leq C$, and $\sum_{i=1}^{n} \alpha_{i} y_{i}=0$. The application of kernel functions for non-linear data makes the algorithm efficient, so that simple hyperplanes are constructed in a space with high dimensions.

In this study, the histogram intersection kernel (Barla, Odone, \& Verri, 2003) is adopted, as defined by Eq. (25), since such a kernel is proposed especially for image classification and it has achieved superior results compared to other kernels. The histogram intersection kernel has been proposed for colour-based image recognition (Barla et al., 2003), whereas in this study it is based on all extracted lesion features, i.e., asymmetry, border, colour and texture:

$K\left(\mathrm{x}_{i}, \mathrm{x}_{j}\right)=\sum_{i=1}^{n} \min \left(x_{i}, x_{j}\right)$.

Here, the classification algorithm is based on supervised learning and the classification process is binary, since the SVM classifier is originally binary. The image classification is divided into two steps: feature classification and skin lesion classification. The feature classification step consists of analysing the following classification processes: (1) region asymmetry (symmetric or asymmetric), (2) border irregularity (regular or irregular), (3) colour uniformity (uniform or non-uniform), and (4) texture irregularity (regular or irregular). Each process takes into account only the features related to the classification goal, i.e., a subset of features. The skin lesion classification step consists of distinguishing the following types of skin lesions: (1) nevus and seborrheic keratosis, (2) nevus and melanoma, and (3) seborrheic keratosis and melanoma. In this case, each classification process considers the entire set of features.

\section{Experimental results and discussion}

In this section, segmentation and classification results are described and discussed. First, the image databases used to evaluate the results are described. Second, the experiments for border detection, regarding the pre-processing, segmentation and postprocessing steps are presented. Finally, the experiments on the feature extraction of skin lesions, which correspond to the lesion's asymmetry, border, colour, and texture, are presented as well as those for lesion classification.

\subsection{Image databases}

The databases used to evaluate the proposed approach are composed of macroscopic images of pigmented skin lesions. Examples of such images are shown in Fig. 1. A great deal of information concerning the diagnosis of the imaged lesions provided by expert dermatologists is available in these databases, including among them, diagnostics on the lesions and their features (i.e., asymmetry, border, colour and texture). All the information contained in the datasets has been used for the development and evaluation of this work.

The used databases have a total of 408 images, which were collected from Loyola University Chicago (Melton \& Swanson, 2012), YSP Dermatology Image Database (Suzumura, 2012), DermAtlas (Cohen \& Lehmann, 2012), DermIS (Diepgen \& Yihune, 2012), Saúde Total (Saúde Total, 2012), Skin Cancer Guide (Skin Cancer Guide, 2012), and Dermnet - Skin Disease Atlas (Campbell Jr., 2012; Chapman, 2012). Of these, 62 images were melanocytic nevi, 86 images were seborrheic keratosis, and 260 images were melanoma. In regard to the asymmetry criterion, the lesions were symmetric in 137 images and in 271 images were asymmetric. In regard to the border criterion, the lesions have regular borders in 77 images and irregular borders in 331 images. In regard to the colour criterion, the lesions present uniform colours in 32 images and nonuniform colours in 376 images. In regard to the texture criterion, the lesions present regular texture in 224 images and in 184 images they present irregular texture. The images of the databases have been resized to $200 \times 200$ pixels to simplify their processing.

\subsection{Border detection}

In order to remove noise and enhance the lesions, an anisotropic diffusion filter was applied to the input images according to the discretization of Eq. (1). The parameters were defined by experimental tests, based on parameters suggested by Barcelos and Pires (2009), with the following values: $\Delta t=0.1, \sigma=1, \lambda=1, k=$ 0.0008 , and $N I=100$. The smoothing results obtained by applying the anisotropic diffusion filter to grey-level images are shown in Fig. 3(a-c). The resultant images in $(\mathrm{d}-\mathrm{f})$ indicate that the filter has successfully reduced the presence of hairs. However, this filter may not remove other artefacts, such as, reflections and shadows.

Afterwards, Chan-Vese's model was applied to segment the smoothed image according to Eq. (4). The parameters were defined by experimental tests, based on the parameters proposed by Chan and Vese (2001): $\mu=0.2, v=0, \lambda_{1}$ and $\lambda_{2}=1, h=1$, $\Delta t=0.1$, and 500 iterations were established for the evolution of the curve. In order to define an appropriate curve $C$, several initial shapes and sizes were tried and visually assessed. A squareshaped curve was defined and positioned close to the image's centre. However, the imaging conditions are usually inconsistent, and the clinical images are acquired from variable distances, implying that the size of the lesions may be different as they are dependent on the distance adopted in the image acquisition. Therefore, two curves, $C_{s}$ and $C_{b}$, with different sizes were established: for small lesions, $C_{s}=40 \times 40$ pixels, and for large lesions, $C_{b}=140 \times 140$ pixels. Examples of the segmentation results obtained by applying the Chan-Vese model to the smoothed images (d-f) are shown in Fig. 3. Although the resultant binary images (g-i) are of good quality, some binary images presented holes in the interior of the seguemented lesion region and/or split regions, which were mainly caused by reflections and shadows.

A morphological filter (Gonzalez \& Woods, 2002) was applied to the segmented binary images to achieve better segmentation results. In order to define an appropriate structuring element $E$, several shapes and sizes were tested. Ellipse-shaped structuring elements with radii $r_{1}, r_{2}=4$, presented the best results according to a visual assessment. The post-processing results obtained by applying the morphological filter to the binary images ( $g-i)$ are shown in Fig. 3. The resultant images $(\mathrm{j}-\mathrm{l})$ confirm the removing of isolated regions and the filling of hole regions, as well as the smoothing of the borders without losing their important characteristics. Afterwards, the borders found were overlapped on the original images $(\mathrm{m}-\mathrm{o})$ based on the post-processing image results $(\mathrm{j}-\mathrm{l})$.

A subjective evaluation (Celebi, Iyatomi, Schaefer, \& Stoecker, 2009) was applied to evaluate the proposed approach, which included a visual assessment of the border detection results by a specialist. The first evaluation analysed whether the lesions were correctly or incorrectly segmented; Fig. 4 includes some example results. The evaluation of the results obtained revealed that the proposed approach is effective in detecting skin lesions and extracting their contours from clinical images. The proposed approach adequately tackled the noisy images. However, some images with low contrast boundaries, shadows and reflections were incorrectly segmented.

The second evaluation compared the segmentation results obtained by the proposed approach against the threshold-based segmentation results achieved by using Otsu's method (Otsu, 1979), since this method has been widely applied in this domain (Abbas et al., 2013a; Celebi et al., 2007b; Norton et al., 2010, 2012). Fig. 5 presents examples of the segmentation results obtained by applying both segmentation methods to the original images (a-e). The 

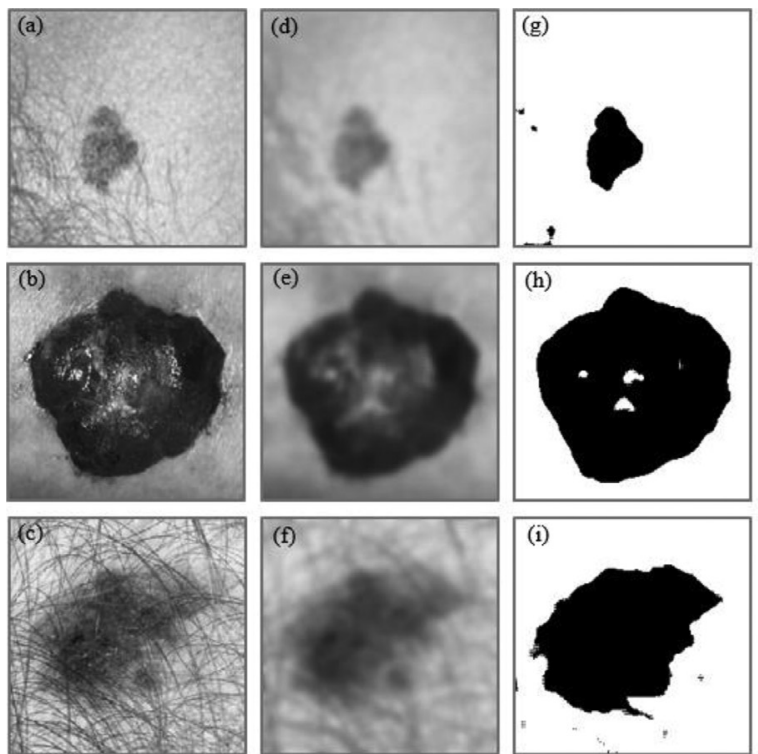
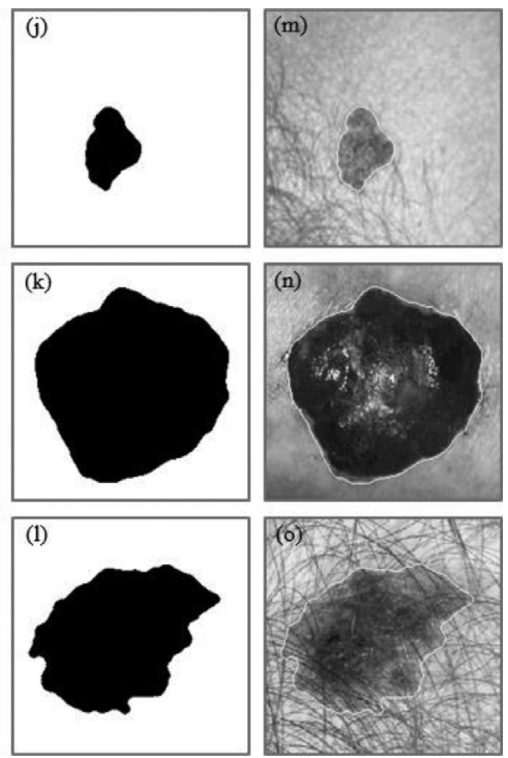

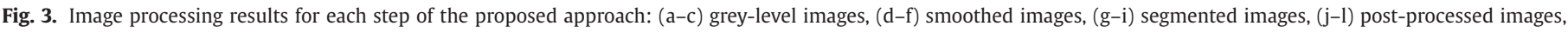
and $(\mathrm{m}-\mathrm{o})$ original images with the detected borders (white contours) overlapped.
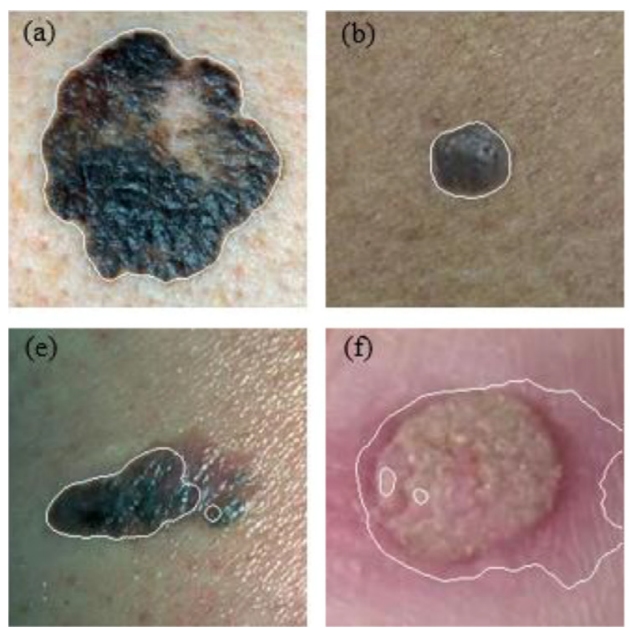
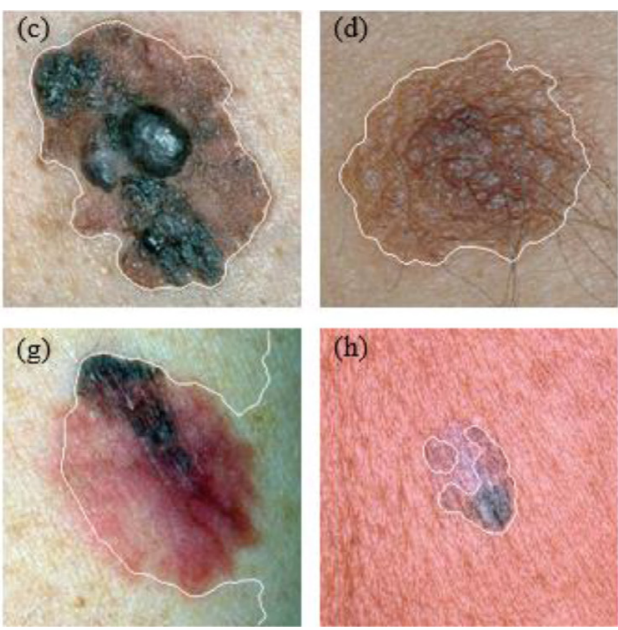

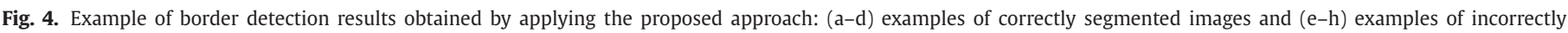
segmented images.

Table 1

Skin lesion segmentation results.

\begin{tabular}{lllll}
\hline Segmentation method & Melanocytic nevus (\%) & Seborrheic keratosis (\%) & Melanoma (\%) & All (\%) \\
\hline Thesholding & 80.65 & 81.40 & 80 & 80.39 \\
Proposed approach & 96.77 & 93.02 & 94.23 & 94.36 \\
\hline
\end{tabular}

evaluation performed on the results obtained revealed that the proposed approach defined the border of the lesion in a more effective way than Otsu's method in several cases. Furthermore, the proposed approach also achieved better results when dealing with images of low contrast, and with shadows and reflections. The percentages of correctly segmented images for both segmentation methods, based on the visual assessment of the resultant borders by a specialist, are shown in Table 1 . It may be seen that the proposed approach obtained significantly superior results compared to the threshold-based method. The quality of the detected borders of the 385 images correctly segmented by the proposed approach was also visually evaluated by the specialist, with $91.43 \%$ of these considered having good quality and the remaining ones having acceptable quality.

\subsection{Skin lesion classification}

In order to differentiate types of skin lesions and to detect their features, several classification experiments were performed. The sets of training and test for the classification process were randomly defined from the available image samples, i.e., from the 385 correctly segmented images. In order to define adequate training sets and test for each classification problem, several sizes for the training set were assessed, with the remaining ones employed as test sets. The size values considered for the training set were $\mathrm{T}_{S}=\{10,20,30,40,50\}$ (in percentage). Each classification model was obtained by applying the SVM classifier (Burges, 1998) by using a histogram intersection kernel (Barla et al., 2003) based on the set or subset of features and on the samples of the training 

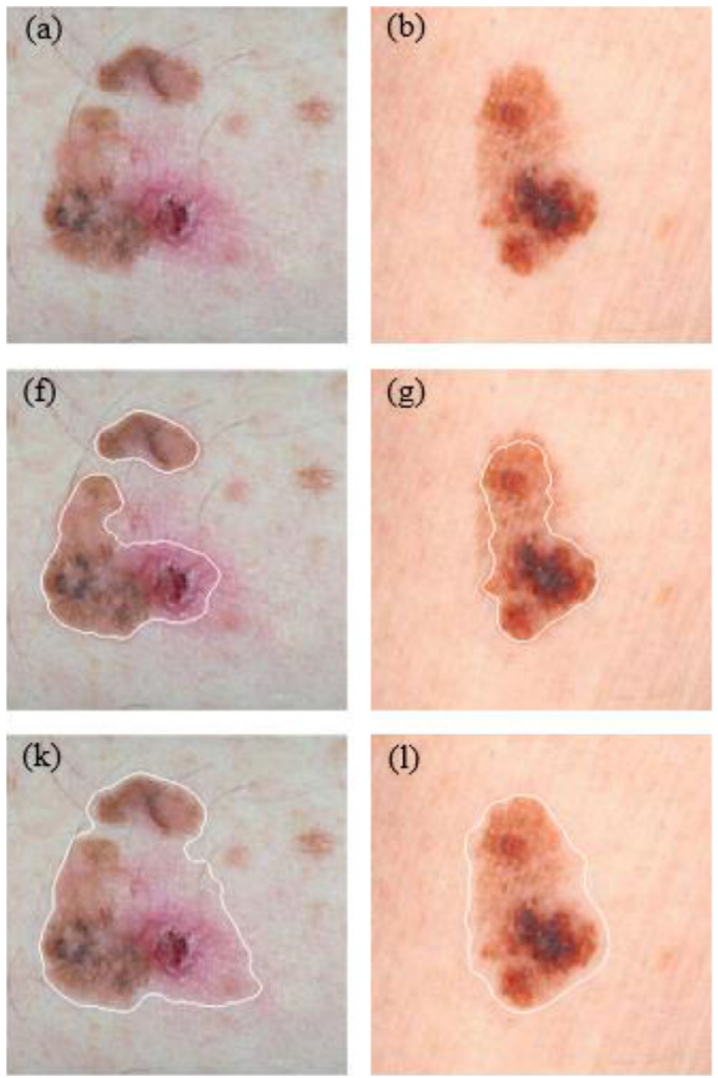
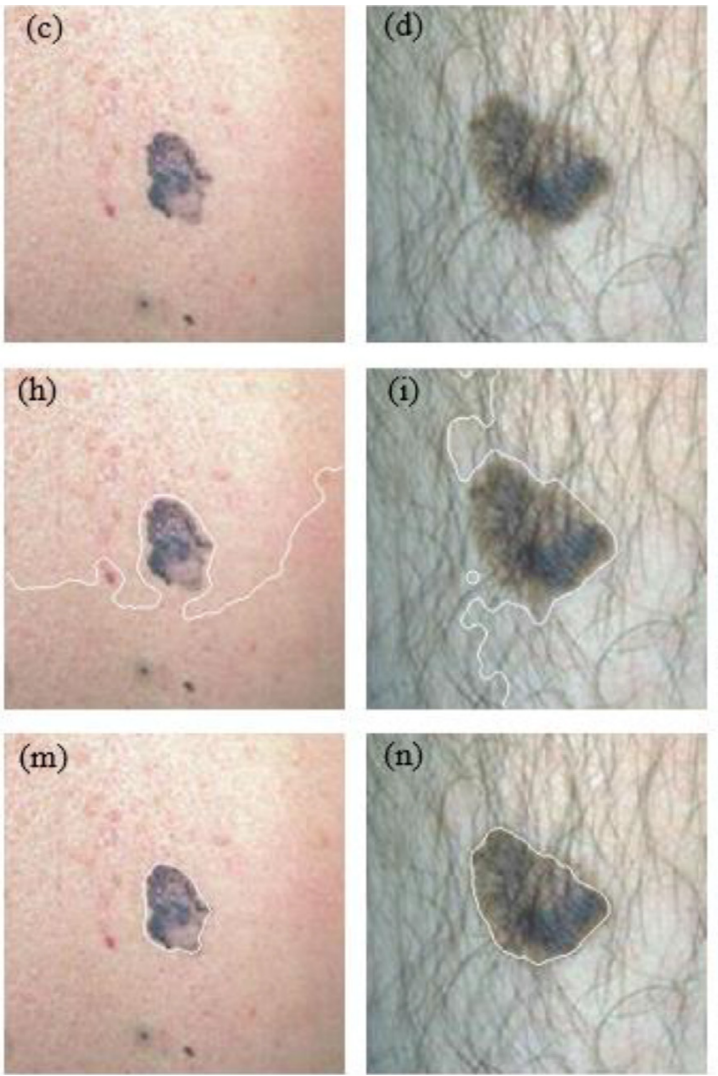
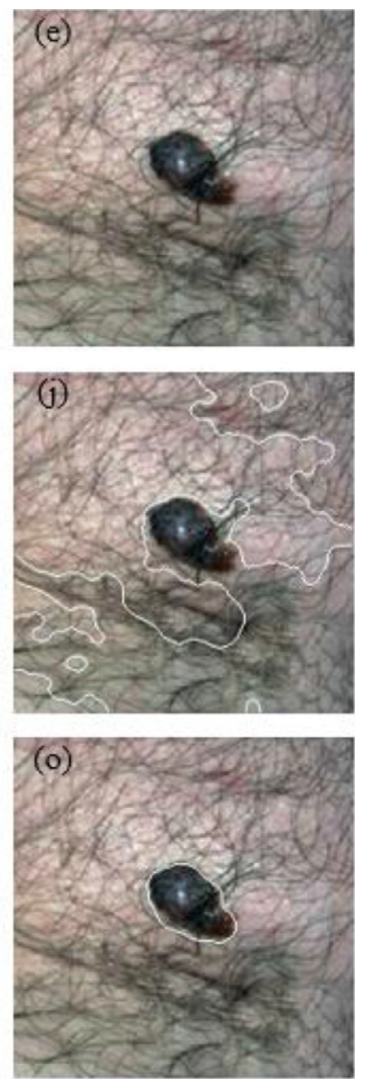

Fig. 5. Comparison of the two segmentation methods: (a-e) original images, (f-j) borders detected by Otsu's method, and (k-o) detected by the proposed approach.

Table 2

Feature classification results of the proposed approach.

\begin{tabular}{|c|c|c|c|c|c|c|}
\hline \multirow[t]{2}{*}{ Classification } & \multicolumn{3}{|c|}{ Radial basis function } & \multicolumn{3}{|c|}{ Histogram intersection } \\
\hline & Class 1 Precision (\%) & Class 2 Precision (\%) & Accuracy (\%) & Class 1 Precision (\%) & Class 2 Precision (\%) & Accuracy (\%) \\
\hline \multirow[t]{2}{*}{ Asymmetry } & Symmetric & Asymmetric & 69.45 & Symmetric & Asymmetric & \\
\hline & 60.71 & 73.62 & & 89.29 & 100 & 96.54 \\
\hline \multirow[t]{2}{*}{ Border } & Regular & Irregular & 81.35 & Regular & Irregular & \\
\hline & 22.86 & 94.30 & & 71.43 & 74.68 & 74.09 \\
\hline \multirow[t]{2}{*}{ Colour } & Uniform & Non-uniform & 73.06 & Uniform & Non-uniform & \\
\hline & 43.75 & 75.71 & & 56.25 & 75.14 & 73.58 \\
\hline \multirow[t]{2}{*}{ Texture } & Regular & Irregular & 62.73 & Regular & Irregular & \\
\hline & 61.69 & 64.10 & & 60.39 & 69.23 & 64.21 \\
\hline
\end{tabular}

set. Afterwards, the samples of the test set were classified based on the classification model and the predicted classes were compared to the known classes. Classification performance metrics, such as the precision for each class and the accuracy for each model, were measured to assess the quality of the results obtained.

The following experiments for feature classification were performed: (1) the first experiment involved asymmetry classification, in which $T_{S}=10$ was considered the best training set, (2) the second experiment comprised the border classification, in which $\mathrm{T}_{S}=$ 50 was considered the best training set, (3) the third experiment comprised the colour classification, in which $\mathrm{T}_{S}=50$ was considered the best training set, and (4) the last experiment was the texture classification, in which $\mathrm{T}_{S}=30$ was considered the best training set. The feature classification results are shown in Table 2. The asymmetry classification obtained good results for both classes. In contrast, the texture and colour feature classifications have not led to good generalization between the classes, whereas border feature classification has resulted in an average distinction between the two classes.
The results obtained for the skin lesion classification are shown in Table 3. The following experiments for skin lesion classification were performed: (1) the first experiment involved classification between nevus and seborrheic keratosis, in which $\mathrm{T}_{S}=40$ was considered the best training set. Although these two types of lesions are benign, the classification model had an average separation between the two classes, (2) the second experiment was determined by the classification between nevus and melanoma, in which $\mathrm{T}_{S}=50$ was considered the best training set. The classification result between these two types of lesion has not been quite expressive, since several samples of the database are composed of skin lesions that do not exactly follow the rule that distinguishes these lesions, and (3) the last experiment was based on the classification between seborrheic keratosis and melanoma, in which $\mathrm{T}_{S}=20$ was considered the best training set. In this case, such lesions are usually too similar, with texture being the main feature used to differentiate them. Therefore, the outcome of the texture classification properly explains why the classification results between seborrheic keratosis and melanoma were not so expressive. 
Table 3

Skin lesion classification results of the proposed approach.

\begin{tabular}{|c|c|c|c|c|c|c|}
\hline \multirow[t]{2}{*}{ Classification } & \multicolumn{3}{|c|}{ Radial basis function } & \multicolumn{3}{|c|}{ Histogram intersection } \\
\hline & Class 1 Precision (\%) & Class 2 Precision (\%) & Accuracy (\%) & Class 1 Precision (\%) & Class 2 Precision (\%) & Accuracy (\%) \\
\hline Nevus-Keratosis (Class 1-Class 2) & 72.22 & 73.33 & 72.84 & 77.78 & 80 & 79.01 \\
\hline Nevus-Melanoma (Class 1-Class 2) & 56.67 & 73.02 & 69.87 & 76.67 & 73.81 & 74.36 \\
\hline Keratosis-Melanoma (Class 1-Class 2) & 60 & 72.64 & 69.73 & 80 & 72.64 & 74.33 \\
\hline
\end{tabular}

The classification results obtained by applying the histogram intersection kernel for the SVM classifier were compared with the results obtained by applying the radial basis function (RBF) kernel (Celebi et al., 2007b; Maglogiannis \& Doukas, 2009; Rahman et al., 2008). The comparison results between the two kernels for both feature and skin lesion classifications are shown in Tables 2 and 3 , respectively. The application of a histogram intersection kernel showed superior performances for the image classifications. Although the border classification by using an RBF kernel had better accuracy than the classification by using a histogram intersection kernel, the precision of the regular border classification was somewhat low (22.86\%). On the other hand, the border classification by using a histogram intersection kernel achieved a more balanced classification result between the regular and irregular classes. In regard to the colour and texture classification, the results were similar for both kernels. In contrast, the asymmetry classification presented significantly superior results. Moreover, the application of a histogram intersection kernel presented much better results for all skin lesion classifications than the RBF kernel.

The proposed approach has been developed using: (1) Matlab 8.4.0.150421 environment for the algorithms of pre-processing, segmentation, post-processing and feature extraction; and (2) Dev$\mathrm{C}++5.11$ environment for the algorithms of texture extraction and classification. The pre-processing step took $63.76 \mathrm{~s}$ in smoothing the 385 images. As to the segmentation step, the algorithm took around $49.12 \mathrm{~min}$ to segment the images. The post-processing step required $5.09 \mathrm{~s}$ to enhance the segmented images. The extraction of the image features from the enhanced images required 1.54 min: asymmetry, $48.65 \mathrm{~s}$; border, $7.35 \mathrm{~s}$; colour, $6.53 \mathrm{~s}$; and texture, $29.44 \mathrm{~s}$. Finally, the classifier required a total of $4.48 \mathrm{~s}$ for the training and testing steps. From these values, which are the average times over 10 runs, one can note that the segmentation step was the most time-consuming; however, the computation time required by this step can be considerably decreased by using optimized $\mathrm{C} / \mathrm{C}++$ implementations. All algorithms were performed on an Intel(R) Core(TM) i5 CPU 650 @ $3.20 \mathrm{GHz} 3.33 \mathrm{GHz}$ with 8 GB of RAM, running Microsoft Windows 7 Professional 64-bits.

\section{Conclusion and future works}

There are several approaches in the literature for pigmented skin lesion classification. Nevertheless, most of the studies involve only dermoscopy images, in which these images may be more difficult to obtain, since they require a dermoscopy device. In contrast, macroscopic images may be obtained using common digital video or image cameras, so that many computational methods to process them become accessible to dermatologists in several regions of the world. Furthermore, the feature classifications in macroscopic images are still little explored in research on automated diagnosis, and most studies do not deal with the classification of all features considered in this paper.

An approach was presented for the segmentation and classification of pigmented skin lesions in macroscopic images. This approach is based on an anisotropic diffusion filter, Chan-Vese's model and an SVM classifier to allow for extracting lesion features and the distinguishing between some types of skin lesions, in order to assist dermatologists in their diagnosis. Asymmetry, border, colour and texture properties were considered for the classification process. Although the proposed approach achieved good segmentation results, mainly with noisy images, it may not perform well on images with too low contrast boundaries, shadows and reflections. Both feature and skin lesion classification presented significant results. However, some classification results were not expressive, e.g., the colour and texture based classifications. Whereas these features were extracted from the original RGB images of the databases, in which some images contain too much hair and too many reflections and shadows. Therefore, such artefacts may harm the assessment of the colour and texture properties of the lesions. In addition, the features of some images of the databases are too heterogeneous for both classes, which can adversely affect the classification results. Unbalanced databases regarding the number of samples for each class may have decreased the accuracy of the classification results, since the classifier tends to be based on classes with the highest occurrence.

In conclusion, future studies regarding the segmentation and classification of pigmented skin lesion images should involve searching for new methods aiming to develop more efficient and effective systems for better computational diagnosis based on macroscopic images. For example, the development of methods for dealing with reflections and shadows may be considered, in order to solve the previously discussed problems concerning the image segmentation step. Other features and types of pigmented skin lesions may also be approached for the purpose of lesions classification from macroscopic images. The skin lesion classification results can be improved using deep learning architectures, since these architectures have presented excellent performances in different applications, including of Computational Vision. From the advantages that these architectures have revealed, one can stress the capacity of learning from large amount of data in an unsupervised way (Bengio, 2009). Therefore, deep learning architectures should be taken into account in future works related to the classification of skin lesions in images.

\section{Acknowledgments}

The first author would like to thank CNPq ("Conselho Nacional de Desenvolvimento Científico e Tecnológico"), in Brazil, for her PhD grant. The authors would also like to thank CAPES ("Coordenação de Aperfeiçoamento de Pessoal de Nível Superior"), in Brazil, for the financial support.

Authors gratefully acknowledge the funding of Project NORTE01-0145-FEDER-000022 - SciTech - Science and Technology for Competitive and Sustainable Industries, cofinanced by "Programa Operacional Regional do Norte" (NORTE2020), through "Fundo Europeu de Desenvolvimento Regional" (FEDER).

Furthermore, the authors thank Dr. Ricardo Baccaro Rossetti, from Derm Clínica's Dermatologist of São José do Rio Preto, in Brazil, for his suggestions and for evaluating the results obtained.

\section{References}

Abbas, Q., Celebi, M. E., \& Garcia, I. F. (2012a). A novel perceptually-oriented approach for skin tumor segmentation. International Journal of Innovative Computing, Information and Control, 8, 1837-1848. 
Abbas, Q., Celebi, M. E., \& García, I. F. (2012b). Skin tumor area extraction using an improved dynamic programming approach. Skin Research and Technology, 18, 133-142.

Abbas, Q., Garcia, I. F., Celebi, M. E., Ahmad, W., \& Mushtaq, Q. (2013a). A perceptually oriented method for contrast enhancement and segmentation of dermoscopy images. Skin Research and Technology, 19, e490-e497.

Abbas, Q., Garcia, I. F., Celebi, M. E., Ahmad, W., \& Mushtaq, Q. (2013b). Unified approach for lesion border detection based on mixture modeling and local entropy thresholding. Skin Research and Technology, 19, 314-319.

Abbasi, N. R., Shaw, H. M., Rigel, D. S., Friedman, R. J., McCarthy, W. H., Osman, I., et al. (2004). Early diagnosis of cutaneous melanoma: Revisiting the ABCD criteria. Jama, 292, 2771-2776.

Al-Akaidi, M. (2004). Fractal speech processing. Cambridge University Press.

Araujo, A.F.D. (2010). Método para extração e caracterização de lesões de pele usando difusão anisotrópica, crescimento de regiões, watersheds e contornos ativos. Unpublished master's thesis, Universidade Estadual Paulista, Instituto de Biociências, Letras e Ciências Exatas, Brasil (in Portuguese).

Barcelos, C. A. Z., Boaventura, M., \& Silva, E. C., Junior (2003). A well-balanced flow equation for noise removal and edge detection. IEEE Transactions on Image Processing, 12, 751-763.

Barcelos, C. A. Z., \& Pires, V. B. (2009). An automatic based nonlinear diffusion equations scheme for skin lesion segmentation. Applied Mathematics and Computation, 215, 251-261.

Barla, A., Odone, F., \& Verri, A. (2003). Histogram intersection kernel for image classification. In International conference on image processing: 3 (pp. 513-516).

Bengio, Y. (2009). Learning deep architectures for AI. Foundations and Trends $®$ in Machine Learning, 2, 1-127.

Beuren, A. T., Janasieivicz, R., Pinheiro, G., Grando, N., \& Facon, J. (2012). Skin melanoma segmentation by morphological approach. In International conference on advances in computing, communications and informatics (pp. 972-978). Chennai, India: ACM.

Burges, C. J. C. (1998). A tutorial on support vector machines for pattern recognition. Data Mining and Knowledge Discovery, 2, 121-167.

Campbell, J. L., Jr. (2012). Dermnet, skin disease atlas. Nevi: Melanoma.

Cavalcanti, P. G., \& Scharcanski, J. (2013). Macroscopic pigmented skin lesion segmentation and its influence on lesion classification and diagnosis. In M. E. Celebi, \& G. Schaefer (Eds.). In Color medical image analysis: Vol. 6 (pp. 15-39). Netherlands: Springer.

Celebi, M. E., Aslandogan, Y. A., Stoecker, W. V., Iyatomi, H., Oka, H., \& Chen, X. (2007a). Unsupervised border detection in dermoscopy images. Skin Research and Technology, 13, 454-462.

Celebi, M. E., Iyatomi, H., Schaefer, G., \& Stoecker, W. V. (2009). Lesion border detection in dermoscopy images. Computerized Medical Imaging and Graphics, 33, 148-153.

Celebi, M. E., Iyatomi, H., Stoecker, W. V., Moss, R. H., Rabinovitz, H. S., Argenziano, G., et al. (2008a). Automatic detection of blue-white veil and related structures in dermoscopy images. Computerized Medical Imaging and Graphics, 32, 670-677.

Celebi, M. E., Kingravi, H. A., Iyatomi, H., Alp Aslandogan, Y., Stoecker, W. V., Moss, R. H., et al. (2008a). Border detection in dermoscopy images using statistical region merging. Skin Research and Technology, 14, 347-353.

Celebi, M. E., Kingravi, H. A., Uddin, B., Iyatomi, H., Aslandogan, Y. A., Stoecker, W. V., et al. (2007b). A methodological approach to the classification of dermoscopy images. Computerized Medical Imaging and Graphics, 31, 362-373.

Celebi, M. E., Wen, Q., Hwang, S., Iyatomi, H., \& Schaefer, G. (2013). Lesion border detection in dermoscopy images using ensembles of thresholding methods. Skin Research and Technology, 19, e252-e258.

Chan, T. F., \& Vese, L. A. (2001). Active contours without edges. IEEE Transactions on Image Processing, 10, 266-277.

Chang, Y., Stanley, R. J., Moss, R. H., \& Van Stoecker, W. (2005). A systematic heuristic approach for feature selection for melanoma discrimination using clinical images. Skin Research and Technology, 11, 165-178.

Chapman, S. (2012). Dermnet, skin disease atlas, benign tumors http://www.dermnet. com/videos/benign-tumors/ Accessed 01.08.2012

Clawson, K. M., Morrow, P., Scotney, B., McKenna, J., \& Dolan, O. (2009). Analysis of pigmented skin lesion border irregularity using the harmonic wavelet transform. In 13th international machine vision and image processing conference (pp. 18-23). IEEE

Cohen, B. A., \& Lehmann, C. U. (2012). Dermatology image atlas. DermAtlas: Johns Hopkins University http://dermatlas.med.jhmi.edu/.

Diepgen, T. L., \& Yihune, G. (2012). Dermatology information system - DermIS. Dermatology Online Atlas http://www.dermis.net/dermisroot/en/home/index.htm Accessed 01.08.2012.

Dobrescu, R., Dobrescu, M., Mocanu, S., \& Popescu, D. (2010). Medical images classification for skin cancer diagnosis based on combined texture and fractal analysis. WISEAS Transactions on Biology and Biomedicine, 7, 223-232.

Filho, M., Ma, Z., \& Tavares, J. M. R. S. (2015). A review of the quantification and classification of pigmented skin lesions: From dedicated to hand-held devices. Journal of Medical Systems, 39, 1-12.

Garnavi, R., Aldeen, M., \& Bailey, J. (2012). Computer-aided diagnosis of melanoma using border- and wavelet-based texture analysis. IEEE Transactions on Information Technology in Biomedicine, 16, 1239-1252.

Gonzalez, R. C., \& Woods, R. E. (2002). Digital image processing (2nd ed.). New Jersey: Prentice Hall.
INCA. (2014). Estimativa 2014: Incidência de câncer no Brasil. Rio de Janeiro: INCA (in Portuguese).

Iyatomi, H., Norton, K., Celebi, M. E., Schaefer, G., Tanaka, M., \& Ogawa, K. (2010). Classification of melanocytic skin lesions from non-melanocytic lesions. In Annual international conference of the IEEE engineering in medicine and biology society (pp. 5407-5410).

Iyatomi, H., Oka, H., Celebi, M. E., Hashimoto, M., Hagiwara, M., Tanaka, M. et al. (2008). An improved Internet-based melanoma screening system with dermatologist-like tumor area extraction algorithm. Computerized Medical Imaging and Graphics, 32, 566-579.

Kass, M., Witkin, A., \& Terzopoulos, D. (1988). snakes: Active contour models. International Journal of Computer Vision, 1, 321-331.

Li, C., Xu, C., Gui, C., \& Fox, M. D. (2010). Distance regularized level set evolution and its application to image segmentation. IEEE Transactions on Image Processing, 19, 3243-3254.

Maglogiannis, I., \& Doukas, C. N. (2009). Overview of advanced computer vision systems for skin lesions characterization. IEEE Transactions on Information Technology in Biomedicine, 13, 721-733.

Melton, J. L., \& Swanson, J. R. (2012). Skin cancer and benign tumor image atlas. Loyola University Dermatology Medical Education, http://www.meddean.luc.edu/ lumen/MedEd/medicine/dermatology/melton/content1.htm Accessed 01.08.2012.

Norton, K.-A., Iyatomi, H., Celebi, M. E., Ishizaki, S., Sawada, M., Suzaki, R., et al. (2012). Three-phase general border detection method for dermoscopy images using non-uniform illumination correction. Skin Research and Technology, $18,290-300$.

Norton, K., Iyatomi, H., Celebi, M. E., Schaefer, G., Tanaka, M., \& Ogawa, K. (2010). Development of a novel border detection method for melanocytic and non-melanocytic dermoscopy images. In Annual International Conference of the IEEE Engineering in Medicine and Biology Society (pp. 5403-5406).

Oliveira, R. B., Filho, M. E., Ma, Z., Papa, J. P., Pereira, A. S., \& Tavares, J. M. R. S. (2016). Computational methods for the image segmentation of pigmented skin lesions: A review. Computer Methods and Programs in Biomedicine, 131, 127-141.

Otsu, N. (1979). A threshold selection method from gray-level histograms. IEEE Transactions on Systems, Man and Cybernetics, 9, 62-66.

Paragios, N., \& Deriche, R. (2002). Geodesic active regions and level set methods for supervised texture segmentation. International Journal of Computer Vision, 46, 223-247.

Rahman, M. M., Bhattacharya, P., \& Desai, B. C. (2008). A multiple expert-based melanoma recognition system for dermoscopic images of pigmented skin lesions. In International Conference on BioInformatics and BioEngineering (pp. 1-6). IEEE.

Roberts, M. E., \& Claridge, E. (2003). An Artificially evolved vision system for segmenting skin lesion images. In R. E. Ellis, \& T. M. Peters (Eds.). In Medical image computing and computer-assisted intervention: Vol. 2878 (pp. 655-662). Berlin Heidelberg: Springer.

Total, Saúde (2012). Câncer da Pele: Fotoproteção, Vida saudável com o sol http: //www.saudetotal.com.br/prevencao/topicos/default.asp Accessed 01.08.2012. (in Portuguese).

Schaefer, G., Rajab, M. I., Celebi, M. E., \& Iyatomi, H. (2011). Colour and contrast enhancement for improved skin lesion segmentation. Computerized Medical Imaging and Graphics, 35, 99-104.

Scharcanski, J., \& Celebi, M. E. (2013). Computer vision techniques for the diagnosis of skin cancer. Berlin Heidelberg: Springer-Verlag.

She, Z., Liu, Y., \& Damatoa, A. (2007). Combination of features from skin pattern and ABCD analysis for lesion classification. Skin Research and Technology, 13, 25-33.

Silveira, M., Nascimento, J. C., Marques, J. S., Marcal, A. R. S., Mendonca, T., Yamauchi, S., et al. (2009). Comparison of segmentation methods for melanoma diagnosis in dermoscopy images. IEEE Journal of Selected Topics in Signal Processing, 3, 35-45.

Skin Cancer Guide. Melanoma. (2012). http://www.skincancerguide.ca/melanoma/ images/melanoma_images.html Accessed 01.08.2012.

Smith, L., \& MacNeil, S. (2011). State of the art in non-invasive imaging of cutaneous melanoma. Skin Research and Technology, 17, 257-269.

Suzumura, Y.. YSP dermatology image database. (2012). http://homepage1.nifty. $\mathrm{com} / \mathrm{ysh} / \mathrm{soft}$ e ysp.htm Accessed 01.08.2012.

Wong, A., Scharcanski, J., \& Fieguth, P. (2011). Automatic skin lesion segmentation via iterative stochastic region merging. IEEE Transactions on Information Technology in Biomedicine, 15, 929-936.

Xu, C., \& Prince, J. L. (1998). Snakes, shapes, and gradient vector flow. IEEE Transactions on Image Processing, 7, 359-369.

Yuksel, M. E., \& Borlu, M. (2009). Accurate segmentation of dermoscopic images by image thresholding based on type-2 fuzzy logic. IEEE Transactions on Fuzzy Systems, 17, 976-982.

Zhang, K., Song, H., \& Zhang, L. (2010). Active contours driven by local image fitting energy. Pattern Recognition, 43, 1199-1206.

Zhou, H., Schaefer, G., Celebi, M. E., Iyatomi, H., Norton, K., Liu, T., \& Lin, F. (2010). Skin lesion segmentation using an improved snake model. In Annual international conference of the engineering in medicine and biology society (pp. 1974-1977). IEEE.

Zhou, H., Schaefer, G., Sadka, A. H., \& Celebi, M. E. (2009). Anisotropic mean shift based fuzzy C-means segmentation of dermoscopy images. IEEE Journal of Selected Topics in Signal Processing, 3, 26-34. 